\title{
MicroRNA expression profiling in the colorectal normal-adenoma-carcinoma transition
}

\author{
JIAN LI*, YUNSHI ZHONG* ${ }^{*}$ SHILUN CAI, PINGHONG ZHOU and LIQING YAO
}

\begin{abstract}
Endoscopy Center and Endoscopy Research Institute, Zhongshan Hospital, Fudan University, Shanghai 200032, P.R. China
\end{abstract}
Received March 25, 2018; Accepted May 17, 2019

DOI: 10.3892/ol.2019.10464

\begin{abstract}
Colorectal adenoma is a major precursor to colorectal cancer. Investigating the alteration of microRNA (miRNA/miR) expression during the progression from normal colorectal tissue to adenoma, and finally to colorectal carcinoma may aid our understanding of the biological mechanisms of colorectal tumorigenesis. In the present study, the miRNA expression profiles of normal colorectal tissue, adenoma and colorectal carcinoma from 6 patients were evaluated using miRNA-sequencing. A total of 334 miRNAs were identified as differentially expressed. It was revealed that 34 miRNAs were upregulated in all 6 patients, including miR-135b-5p, miR-18a-5p and miR-29b-3p, and 28 miRNAs were downregulated, including miR-1-3p, miR-338-3p and miR-218-5p. Using bioinformatic analysis, the potential target genes of these 62 miRNAs were predicted and found to be enriched in 'transcription, DNA-dependent (GO:0006351)', 'signal transduction (GO:0007165)', 'small molecule metabolic process (GO:0044281)' 'PI3K/AKT signaling pathway (path ID:04151)' and 'MAPK signaling pathway (path ID:04010)'. The miRNA expression profiles identified in the present study may extend our understanding of the molecular mechanisms underlying colorectal tumorigenesis and promote novel perspectives for prevention, diagnosis and treatment.
\end{abstract}

\section{Introduction}

As one of the most common forms of cancer, colorectal cancer is the third most frequent cause of cancer-related mortality worldwide, accounting for $\sim 1.36$ million new cases and 0.69 million estimated mortalities each year (1). Colorectal adenoma is a major precursor to colorectal cancer, with an

Correspondence to: Dr Liqing Yao, Endoscopy Center and Endoscopy Research Institute, Zhongshan Hospital, Fudan University, 180 Fenglin Road, Shanghai 200032, P.R. China

E-mail: yao.liqing@zs-hospital.sh.cn

${ }^{*}$ Contributed equally

Key words: colorectal cancer, colorectal adenoma, microRNA, microRNA-sequencing, bioinformatic analysis estimated $5 \%$ of adenomas developing into carcinomas $(2,3)$. MicroRNAs (miRNAs/miRs) are small, non-coding RNAs that post-transcriptionally modulate the stability of messenger RNAs to regulate gene expression and serve crucial roles in colorectal tumorigenesis (4). Therefore, clarifying the alteration of miRNA expression profiles in the progression from normal colorectal tissue to adenoma, and finally to carcinoma, is essential for an improved understanding of the biological mechanisms of colorectal tumorigenesis.

Global expression analysis based on high-throughput data has become a powerful technique in miRNA analysis (5). In the present study, using miRNA-sequencing, the miRNA expression profiles in matching normal colorectal tissue, adenoma and carcinoma samples from patients were detected. Critical miRNAs that may be involved in cancer progression were investigated, and their biological functions were assessed through comprehensive bioinformatic analysis. The present results may facilitate a better understanding of the roles of miRNAs in colorectal tumorigenesis and promote novel strategies for the prevention and treatment of colorectal carcinoma.

\section{Materials and methods}

Ethics statements and patient selection. The present study was approved by The Ethics Committee of Zhongshan Hospital, Fudan University (Shanghai, China). Written informed consent was obtained from the 6 male patients with colorectal adenoma and carcinoma and three adjacent samples were collected using colonoscopy from each patient with normal mucosa, adenoma and carcinoma between January 2014 and July 2014. The age of the patients ranged between 49-64 years (mean age \pm standard deviation, $57.67 \pm 6.83$ years).

RNA isolation. Total RNA was extracted from tissue samples using the RNAprep Pure kit (For Tissue) (Tiangen Biotech Co., Ltd.) following the manufacturer's protocol. The concentration and purity of RNA samples were determined by absorbance detection and electrophoresis.

miRNA-sequencing. The sequencing libraries were constructed according to the protocol for the Illumina small RNA sample preparation kit (6). Sequencing was performed on the Illumina HiSeq 2000 sequencer (Illumina, Inc.). Library construction and sequencing were performed at the Genergy Biotech 
Co., Ltd. miRNA expression was analyzed by miRdeep version 2.0.0.7 (6).

Screening for differentially expressed miRNAs. ANOVA and least significant difference post hoc test were performed in GraphPad Prism version 5 (GraphPad Software, Inc.) to compare the mean differences of the expression among the three groups in normal colorectal tissues, colorectal adenomas and tumor tissues. miRNAs with $\mathrm{P}<0.05$ and fold-change $>2$ were considered significantly altered. miRNAs among all 6 patients that were consistently and significantly increased or decreased in adenoma and cancer were identified using an FDR cutoff value of 0.05 and absolute $\log 2$-fold change greater than 1 , and selected for the next stage of analysis. Hierarchical clustering was performed to further sort the selected differentially expressed miRNAs with similar expression patterns, using Gene Cluster 3.0 with the following settings: Distance metric, Pearson correlation; linkage rule, average linkage (bonsai.hgc.jp/ mdehoon/software/cluster/software.htm).

Prediction of miRNA target genes. The target genes of these miRNAs were predicted using TargetScan (www.targetscan. org) (7) and miRanda (www.microrna.org/microrna/home. do) (8) databases. The overlapping predictions obtained from the two databases were considered as potential target genes.

Gene ontology (GO) and Kyoto encyclopedia of genes and genomes (KEGG) analyses. GO and KEGG analyses were applied to analyze the main functions of the target genes and identify the significant pathways. The GO (geneontology. org) (9) and KEGG (www.genome.jp/kegg) (10) databases were used as previously reported $(5,11)$. Enrichment analysis provided a measure of the significance of the GO and KEGG pathways. A threshold of $\mathrm{P}<0.05$ indicated statistical significance.

\section{Results}

Identification of differentially expressed miRNAs. Microarray analysis revealed 334 differentially expressed miRNAs in one normal colorectal, adenoma and carcinoma group compared with the other two groups. Among them, 34 miRNAs were consistently upregulated in tissue undergoing colorectal adenoma-carcinoma transition, including miR-135b-5p, miR-18a-5p and miRNA-29b-3p (the three with the largest fold changes), while 28 miRNAs were consistently downregulated, including miR-1-3p, miR-338-3p and miR-218-5p (the three with the largest fold changes) (Fig. 1). The results of hierarchical cluster analysis of the miRNAs with the largest up and downregulated changes are shown in Fig. 2.

The target genes of these 62 miRNAs were predicted using the miRNA target site databases TargetScan and miRanda, and the overlapping results are listed in Table SI, including 3,715 genes.

Enriched GO terms. Using GO analysis, it was found that the potential target genes of the consistently upregulated miRNAs were significantly enriched in 561 GO terms, while which of consistently downregulated miRNAs were significantly enriched in $475 \mathrm{GO}$ terms. The potential targets of differentially expressed miRNAs were mainly enriched in transcription regulation, signaling and metabolism of biological process, extracellular matrix (ECM), and signal transducer activity of molecular function during colorectal tumorigenesis, such as 'transcription, DNA-dependent (GO:0006351)', 'signal transduction (GO:0007165)', 'small molecule metabolic process (GO:0044281)', 'apoptotic process (GO:0006915)', 'cell adhesion (GO:0007155)' and 'protein transport (GO:0015031)', consistent with the proliferative and invasive capacity of colorectal cancer cells (Fig. 3). In addition, other GO functions associated with target genes included 'activation of MAPK activity (GO:0000187)', 'cell cycle (GO:0007049)', 'cell-cell signaling (GO:0007267)','cell differentiation (GO:0030154)' and 'protein ubiquitination (GO:0016567)'.

Significant KEGG pathways. Using KEGG analysis, it was found that the potential target genes of upregulated miRNAs were significantly enriched in 135 signaling pathways, while those of downregulated miRNAs were significantly enriched in 106 signaling pathways. The majority of these signaling pathways overlapped. The results revealed that potential target genes of the upregulated and downregulated miRNAs are involved in the 'MAPK signaling pathway', which is consistent with the GO analysis (Fig. 4). Target genes were also significantly enriched in the 'PI3K/AKT signaling pathway', 'focal adhesion' and the 'Hippo signaling pathway.'

\section{Discussion}

In the present study, high throughput screening of miRNA expression profiles was performed by sequencing miRNA from adjacent non-tumor, colorectal adenoma and carcinoma tissue samples from the same patient. Careful screening revealed 334 miRNAs as significantly differentially expressed in the three tissues types. These differentially expressed miRNAs may be promising potential diagnostic markers for the progression of colorectal cancer. In support of these findings, a study by Slattery et al (12) also identified $~ 600$ differentially expressed miRNAs in colorectal carcinoma progression using an Agilent microarray platform (12).

In the present study, the potential function of the 62 miRNAs which were consistently upregulated or downregulated during the progression from normal to adenoma, and subsequently to carcinoma, was investigated. It was revealed that miR-135b-5p, miR-18a-5p and miR-29b-3p were consistently upregulated during this transition. Several studies have confirmed the overexpression of miR-135b-5p in colorectal tumors (13-15). Valeri et al (13) reported that miR-135b-5p overexpression was triggered in mice and humans by loss of adenomatous polyposis coli, PTEN/PI3K pathway deregulation and overexpression of proto-oncogene tyrosine-protein kinase $\mathrm{Src}$, and it was also demonstrated that miR-135b-5p promoted tumor transformation and progression. Zhang et al (14) and Liu et al (16) reported that miR-135b-5p acted as an upstream factor of the PI3K/AKT pathway, while the results presented in this study demonstrated that the PI3K/AKT pathway was enriched for targets 


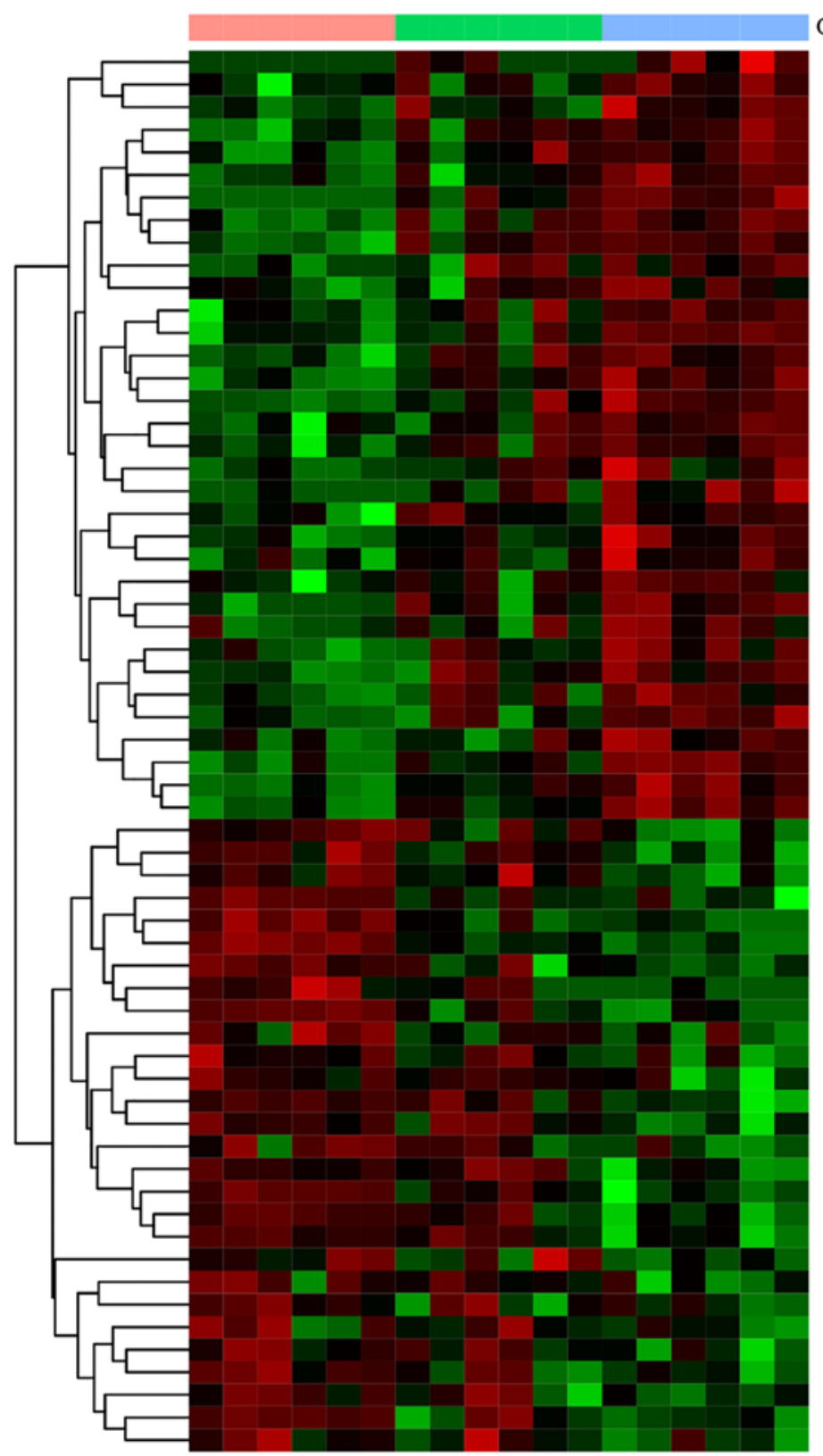

Group

miR-4780

miR-34a-3p

miR-34a-5p

miR-183-5p

miR-18a-5p

miR-29-3p

miR-29b-3p
miR-549a

miR-135b-3p

miR-135b-5p

miR-93-3p

miR-221-3p

miR-203a-3p

miR-203a-5p

miR-3200-3p

miR-339-5p

miR-425-3p

miR-1268a

miR-1268b

miR-6854-5

miR-6854-5p

miR-320b

miR-320c

miR-7641

miR-6723-5p

$\operatorname{miR}-4454$
miR-107

miR-106b-3p

miR-103a-3p

miR-200a-5p

miR-491-5p

miR-19b-1-5p

miR-19b-3p

miR-99a-5

miR-125a-5p

miR-145-3p

miR-9-5p

miR-124-3p

miR-137

miR-218-5p

miR-216b-5p

miR-26a-5p

miR-146b-5p

miR-126-5p
miR-126-3p

miR-561-5p

miR-100-3p

miR-146b-3p

miR-299-5p

miR-539-3p

miR-1-3p

miR-136-3p

miR-381-3p

miR-589-5p

miR-659-5p

miR-1468-5p

let-7e-3p

miR-151b

miR-136-5p

let-7b-3p

miR-338-3p

miR-28-5p

Figure 1. Cluster analysis of the 34 consistently upregulated miRNAs and the 28 consistently downregulated miRNAs in normal colorectal tissue, adenoma and carcinoma samples. Red represents upregulation of miRNAs, and green represents downregulation of miRNAs. miRNA/miR, microRNA.

of differentially expressed miRNAs. Yau et al (17) reported that miR-18a-5p expression was significantly increased in tumor tissues and stool samples of patients with colorectal carcinoma, and that it may serve as a potential biomarker for a non-invasive diagnosis of this cancer. In the present study, miR-29b-3p was significantly upregulated in colorectal tumors. However, Inoue et al (18) reported that miR-29b-3p expression was significantly downregulated in tumor tissue compared with normal mucosa, and that it was an independent positive prognostic factor of disease-free survival. Wang et al (19) reported that miR-29b-3p suppressed tumor growth and metastasis in colorectal cancer by downregulating T-cell lymphoma invasion and metastasis 1 expression and inhibiting epithelial to mesenchymal transition. Therefore, further study is required to clarify the impact of altered miR-29b-3p expression in colorectal tumors.

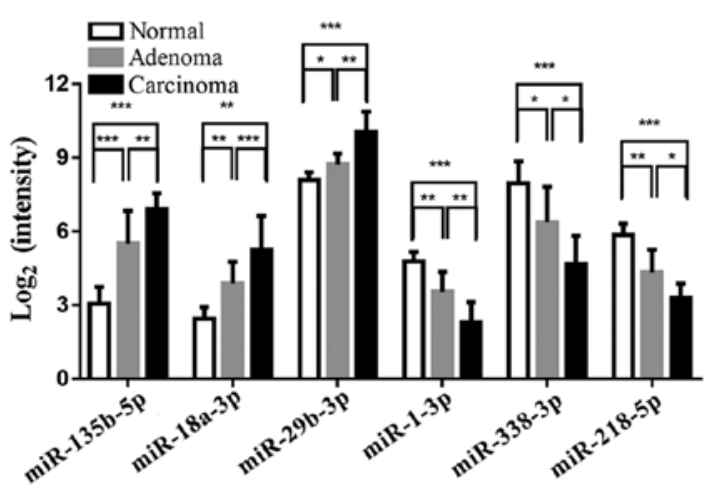

Figure 2. Expression of the three most upregulated miRNAs (miR-135b-5p, miR-18a-5p and miR-29b-3p) and the three most downregulated miRNAs (miR-1-3p, miR-338-3p and miR-218-5p) in the colorectal normal-adenoma-carcinoma transition, as calculated from miRNA sequencing data. miRNA/miR, microRNA. ${ }^{*} \mathrm{P}<0.05,{ }^{* *} \mathrm{P}<0.01$ and ${ }^{* * *} \mathrm{P}<0.001$. 
A

Targetgene Sig GO

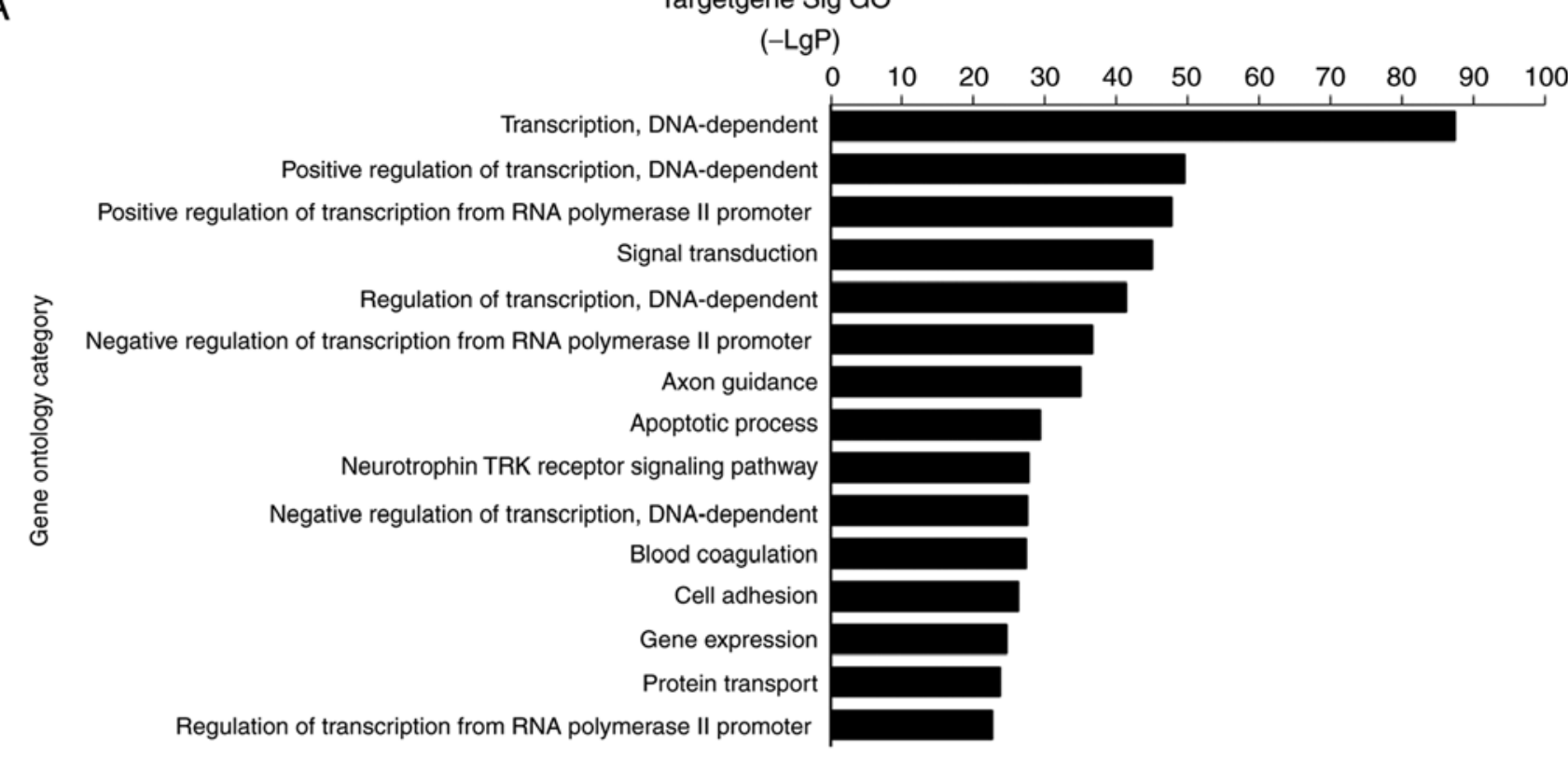

B

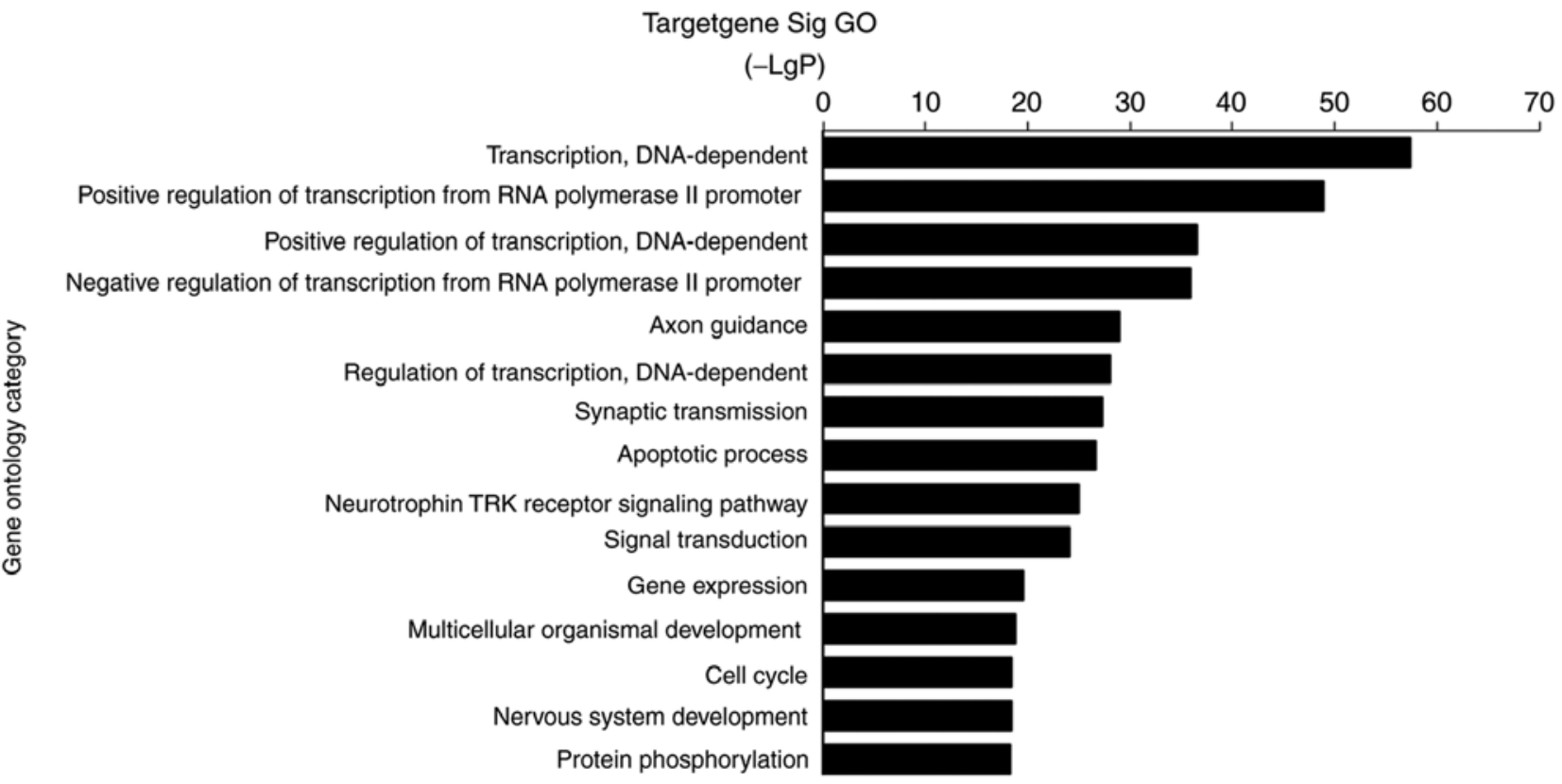

Figure 3. GO analysis. (A) The 15 most significantly upregulated GO terms, and (B) 15 most significantly downregulated GO terms in the colorectal normal-adenoma-carcinoma transition. GO, Gene Ontology; Sig, significant; -LgP, - $\log 10$ (P-value).

The present study also revealed that miR-1-3p, miR-338-3p and miR-218-5p were significantly downregulated throughout the transition from normal tissue to adenoma and then carcinoma. A recent meta-analysis of The Cancer Genome Atlas and Gene Expression Omnibus data performed by Wang et al (20) demonstrated significant miR-1-3p downregulation in colorectal cancer. Specifically, miR-1-3p was found to suppress tumor proliferation via the mothers against decapentaplegic homolog 3, hypoxia-inducible factor 1, and MAPK and PI3K pathways $(21,22)$. Sun et al (23) reported that miR-338-3p expression was reduced in colorectal cancer and was negatively correlated with advanced TNM stage and local invasion. It was also reported that miRNA-338-3p suppressed cell proliferation of human colorectal carcinoma by targeting the smoothened gene (24). Furthermore, several studies reported miR-218-5p downregulation in colorectal cancer, which exerted its tumor suppressor effects via the PI3K/AKT pathway, diphthamide biosynthesis 1 and metastasis-associated in colon cancer 1 , among others (25-28).

Based on the present results, the predicted target genes of differentially expressed miRNAs were enriched in cell cycle, cell adhesion and the PI3K and MAPK signaling pathways. The majority of these GO terms and KEGG pathways have been demonstrated to serve significant roles in colorectal tumorigenesis. Sun et al (29) found that the MAPK pathway was associated with cell proliferation, differentiation, migration, senescence and apoptosis in colorectal carcinoma. Bogaert and Prenen (30) concluded the PI3K/AKT, WNT, p53, receptor tyrosine 
A

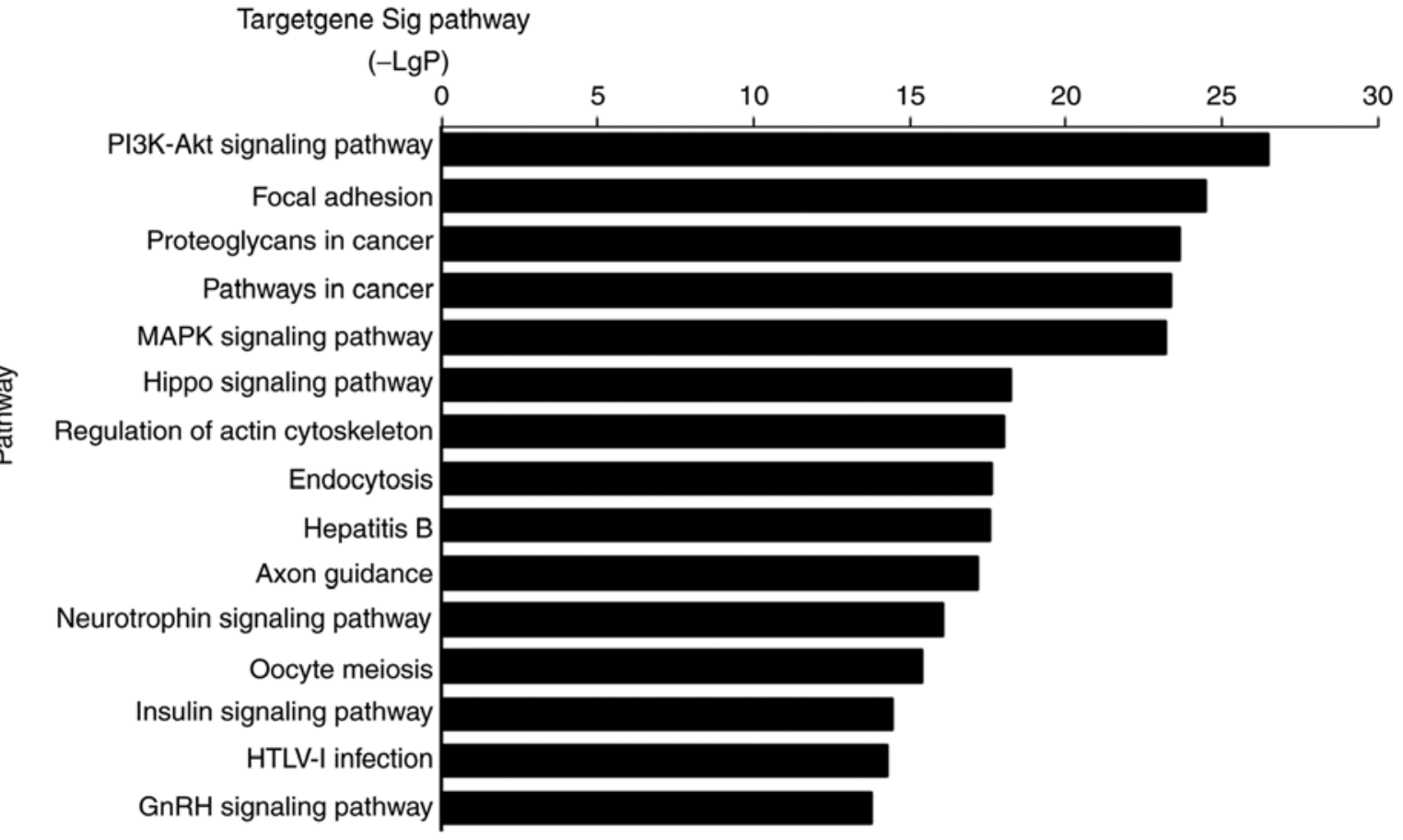

B

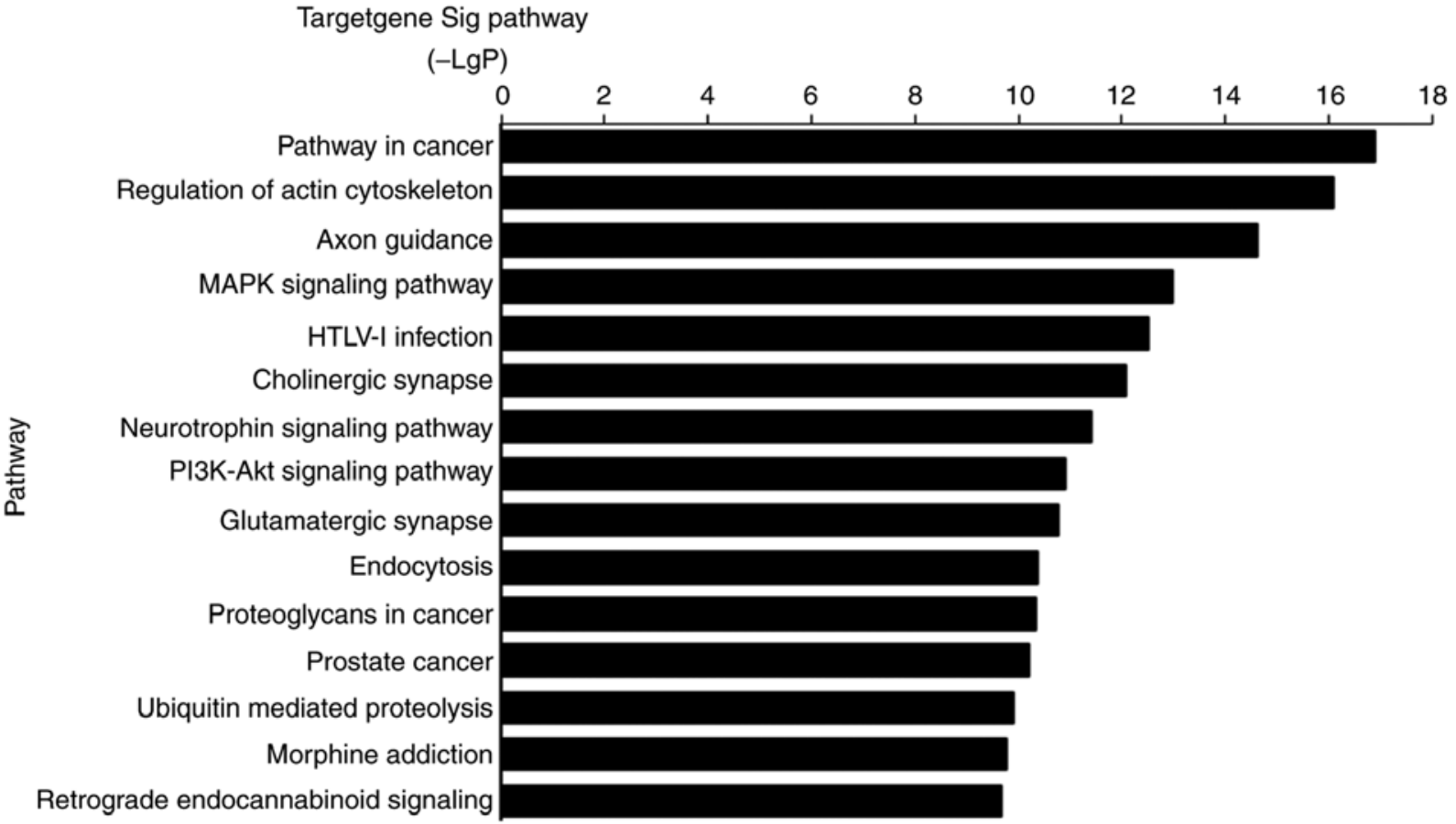

Figure 4. KEGG pathway analysis. (A) The 15 most significantly upregulated KEGG pathways, and (B) 15 most significantly downregulated KEGG pathways in the colorectal normal-adenoma-carcinoma transition. KEGG, Kyoto Encyclopedia of Genes and Genomes; Sig, significant; -LgP, -log10 (P-value).

kinase/RAS GTPase and transforming growth factor $\beta$ pathways to be the five pathways frequently altered in colorectal cancer. Notably, the predicted target genes identified in the present study were also enriched in the majority of these pathways.

It should be noted that there are several limitations to the present study. First, only samples from 6 patients were used. Second, the expression of miRNAs was not validated by other methods such as quantitative PCR. Third, the majority of the results were based on bioinformatics analysis and require further validation.
In the present study, a high-throughput screening of miRNA expression profiles in colorectal cancer development was performed by sequencing miRNA from adjacent normal, colorectal adenoma and carcinoma tissues. The miRNAs that were consistently and significantly upregulated or downregulated were selected, and their biological targets were predicted using bioinformatics analysis. These miRNAs may be promising diagnostic biomarkers and potential treatment targets in colorectal carcinoma. These findings should be further validated in future experiments. 


\section{Acknowledgements}

Not applicable.

\section{Funding}

The present study was supported by The Medical Leading Project of Shanghai Municipal Science and Technology Committee (grant nos. 16DZ2280900 and 19DZ2280100).

\section{Availability of data and materials}

The datasets used and/or analyzed during the present study are available on reasonable request from the corresponding author on reasonable request.

\section{Authors' contributions}

LY, YZ and PZ designed the study. JL and YZ wrote the manuscript. JL and SC conducted the experiments and analyzed the data. All authors read and approved the final manuscript.

\section{Ethics approval and consent to participate}

The present study was approved by The Ethics Committee of Zhongshan Hospital, Fudan University (Shanghai, China) and conducted according to their guidelines and regulations.

\section{Patient consent for publication}

All participants provided informed consent.

\section{Competing interests}

The authors declare that they have no competing interests.

\section{References}

1. Torre LA, Bray F, Siegel RL, Ferlay J, Lortet-Tieulent J and Jemal A: Global cancer statistics, 2012. CA Cancer J Clin 65 87-108, 2015.

2. Muto T, Bussey HJ and Morson BC: The evolution of cancer of the colon and rectum. Cancer 36: 2251-2270, 1975.

3. Shinya H and Wolff WI: Morphology, anatomic distribution and cancer potential of colonic polyps. Ann Surg 190: 679-683, 1979

4. Masuda T, Hayashi N, Kuroda Y, Ito S, Eguchi H and Mimori K: MicroRNAs as biomarkers in colorectal cancer. Cancers (Basel) 9: 124, 2017.

5. Zhan C, Yan L, Wang L, Jiang W, Zhang Y, Xi J, Jin Y, Chen L, Shi Y, Lin Z and Wang Q: Landscape of expression profiles in esophageal carcinoma by the cancer genome Atlas data. Dis Esophagus 29: 920-928, 2016.

6. Friedlander MR, Chen W, Adamidi C, Maaskola J, Einspanier R, Knespel S and Rajewsky N: Discovering microRNAs from deep sequencing data using miRDeep. Nat Biotechnol 26: 407-415, 2008.

7. Agarwal V, Bell GW, Nam JW and Bartel DP: Predicting effective microRNA target sites in mammalian mRNAs. Elife: Aug 12, 2015. doi: 10.7554/eLife.05005.

8. Betel D, Koppal A, Agius P, Sander C and Leslie C: Comprehensive modeling of microRNA targets predicts functional non-conserved and non-canonical sites. Genome Biol 11: R90, 2010.

9. The Gene Ontology Consortium: Expansion of the Gene Ontology knowledgebase and resources. Nucleic Acids Res 45: D331-D338, 2017.
10. Kanehisa M, Furumichi M, Tanabe M, Sato Y and Morishima K: KEGG: New perspectives on genomes, pathways, diseases and drugs. Nucleic Acids Res 45: D353-D361, 2017.

11. Yan L, Zhan C, Wu J and Wang S: Expression profile analysis of head and neck squamous cell carcinomas using data from The Cancer Genome Atlas. Mol Med Rep 13: 4259-4265, 2016.

12. Slattery ML, Herrick JS, Pellatt DF, Stevens JR, Mullany LE, Wolff E, Hoffman MD, Samowitz WS and Wolff RK: MicroRNA profiles in colorectal carcinomas, adenomas and normal colonic mucosa: Variations in miRNA expression and disease progression. Carcinogenesis 37: 245-261, 2016.

13. Valeri N, Braconi C, Gasparini P, Murgia C, Lampis A, Paulus-Hock V, Hart JR, Ueno L, Grivennikov SI, Lovat F, et al: MicroRNA-135b promotes cancer progression by acting as a downstream effector of oncogenic pathways in colon cancer. Cancer Cell 25: 469-483, 2014.

14. Zhang J, Raju GS, Chang DW, Lin SH, Chen Z and Wu X: Global and targeted circulating microRNA profiling of colorectal adenoma and colorectal cancer. Cancer 124: 785-796, 2018.

15. Jia L, Luo S, Ren X, Li Y, Hu J, Liu B, Zhao L, Shan Y and Zhou H: miR-182 and miR-135b mediate the tumorigenesis and invasiveness of colorectal cancer cells via targeting ST6GALNAC2 and PI3K/AKT pathway. Dig Dis Sci 62: 3447-3459, 2017.

16. Liu B, Liu Y, Zhao L, Pan Y, Shan Y, Li Y and Jia L: Upregulation of microRNA-135b and microRNA-182 promotes chemoresistance of colorectal cancer by targeting ST6GALNAC2 via PI3K/AKT pathway. Mol Carcinog 56: 2669-2680, 2017.

17. Yau TO, Wu CW, Dong Y, Tang CM, Ng SS, Chan FK, Sung JJ and Yu J: microRNA-221 and microRNA-18a identification in stool as potential biomarkers for the non-invasive diagnosis of colorectal carcinoma. Br J Cancer 111: 1765-1771, 2014.

18. Inoue A, Yamamoto H, Uemura M, Nishimura J, Hata $T$, Takemasa I, Ikenaga M, Ikeda M, Murata K, Mizushima T, et al: MicroRNA-29b is a novel prognostic marker in colorectal cancer. Ann Surg Oncol 22 (Suppl 3): S1410-S1418, 2015.

19. Wang B, Li W, Liu H, Yang L, Liao Q, Cui S, Wang H and Zhao L: miR-29b suppresses tumor growth and metastasis in colorectal cancer via downregulating Tiam 1 expression and inhibiting epithelial-mesenchymal transition. Cell Death Dis 5: e1335, 2014.

20. Wang JY, Huang JC, Chen G and Wei DM: Expression level and potential target pathways of miR-1-3p in colorectal carcinoma based on 645 cases from 9 microarray datasets. Mol Med Rep 17: 5013-5020, 2018.

21. Xu W, Zhang Z, Zou K, Cheng Y, Yang M, Chen H, Wang H, Zhao J, Chen P, He L, et al: MiR-1 suppresses tumor cell proliferation in colorectal cancer by inhibition of Smad3-mediated tumor glycolysis. Cell Death Dis 8: e2761, 2017.

22. Xu L, Zhang Y, Wang H, Zhang G, Ding Y and Zhao L: Tumor suppressor miR-1 restrains epithelial-mesenchymal transition and metastasis of colorectal carcinoma via the MAPK and PI3K/AKT pathway. J Transl Med 12: 244, 2014.

23. Sun K, Deng HJ, Lei ST, Dong JQ and Li GX: miRNA-338-3p suppresses cell growth of human colorectal carcinoma by targeting smoothened. World J Gastroenterol 19: 2197-2207, 2013.

24. Sun K, Su G, Deng H, Dong J, Lei S and Li G: Relationship between miRNA-338-3p expression and progression and prognosis of human colorectal carcinoma. Chin Med J (Engl) 127: 1884-1890, 2014

25. Liu M, Yin K, Guo X, Feng H, Yuan M, Liu Y, Zhang J, Guo B, Wang C, Zhou G, et al: Diphthamide biosynthesis 1 is a novel oncogene in colorectal cancer cells and is regulated by MiR-218-5p. Cell Physiol Biochem 44: 505-514, 2017.

26. Ilm K, Fuchs S, Mudduluru G and Stein U: MACC1 is post-transcriptionally regulated by miR-218 in colorectal cancer. Oncotarget 7: 53443-53458, 2016.

27. Li PL, Zhang X, Wang LL, Du LT, Yang YM, Li J and Wang CX: MicroRNA-218 is a prognostic indicator in colorectal cancer and enhances 5-fluorouracil-induced apoptosis by targeting BIRC5. Carcinogenesis 36: 1484-1493, 2015.

28. Zhang X, Shi H, Tang H, Fang Z, Wang J and Cui S: miR-218 inhibits the invasion and migration of colon cancer cells by targeting the PI3K/Akt/mTOR signaling pathway. Int J Mol Med 35: 1301-1308, 2015.

29. Sun Y, Liu WZ, Liu T, Feng X, Yang N and Zhou HF: Signaling pathway of MAPK/ERK in cell proliferation, differentiation, migration, senescence and apoptosis. J Recept Signal Transduct Res 35: 600-604, 2015.

30. Bogaert J and Prenen H: Molecular genetics of colorectal cancer. Ann Gastroenterol 27: 9-14, 2014. 